\title{
GUID for Mobility First Architecture Supporting IoUT
}

\author{
Kalyani Muppalla, Nam-Yeol Yun, Soo-Hyun Park ${ }^{\dagger}$ and Changhwa Kim \\ ${ }^{\dagger}$ Ubiquitous System Lab., Graduate School of Business IT \\ Kookmin UniversitySeoul, South Korea \\ Underwater Sensor Network Research Center \\ Gangneung-Wonju National University, Wonju, South Korea \\ \{mkalyani, anuice, shpark21\}@kookmin.ac.kr ${ }^{\dagger}$, kch@gwnu.ac.kr
}

\begin{abstract}
The Mobility First network architecture is introduced to differentiate the human readable names with corresponding Global Unique Identifiers (GUIDs), and the dynamic network address locators. The human readable name can be managed and assigned to a unique GUID by Name Certification Services (NCSs). GUIDs assigned to network objects (Things) are mapped to a set of network addresses (NAS). Delay andDisruption Tolerant Network (DTN) is used in the Mobility First for efficient communication. GUIDs are assigned to Internet of Things (IoT) and Imternet of Underwater Things (IoUT). Underwater things can collect the underwater data using Sensors, underwater modem, underwater Medium Access Control (MAC) board and transducer etc. Internet of Underwater Things (IoUT) can comminieate the changes in the ocean environment to the offshore IoT. Underwater things lare constructuve in offshore investigation, disaster anticipation, data gathering, assisted navigation, pollution checking and strategic inspection. To achieve these features, this paper proposes architecture with three layers. They are IoUT layer, DTR communicationlayer and IoT layer. IoUT layer gathers the information with the help of underwater things. DTN communication layer sends this information to IoT layer with help of Global Name Resolution Service (GNRS). Dynamic binding of names to address is done by GNRS. By using IoT layer ocean data can be broadcasted in different applications like scientific applications, military applications, industrial applications and clvilian applications. Results are shown by using the GUID accessing based on popularity.
\end{abstract}

Keywords: Internet of Underwater Things; Delay Tolerant Network; Underwater Things; Global Unique Identifier; Global Name Resolution Service; Mobility First; Internet of Things.

\section{Introduction}

Mobility First network architecture is a part of future internet architecture program and it is presently under development. This architecture is planned to separate human readable names, the corresponding Global Unique Identifiers (GUIDs), and the dynamic network address locators [4]. The human readable names are assigned to a unique GUID by Name Certification Services (NCSs). GUID space is huge, so the probability of collisions is negligible. For this reason no coordination is required between NCS providers. GUIDs which are assigned to network objects will be mapped to a set of Network Addresses (NAs).

GUIDs are assigned to underwater things for efficient communication. Underwater things are used in offshore investigation, disaster prevention, data collection, assisted navigation, pollution checking and strategic inspection. Underwater thing outfitted with sensors, will allow the examination of underwater resources [6] and gathering of technical

$\dagger$ Corresponding author 
data in collaborative monitoring operation. Internet of Underwater Things (IoUT) [3] find the changes in the ocean environment and communicate to Internet of Things (IoT) [5]. The changes send to the offshore IoT with the help of Mobility First. Global Name Resolution Service (GNRS) is used for dynamic binding of names to address. Delay and Disruption Tolerant Network (DTN) is used to communicate between the underwater acoustic sensor network and internet with help of Mobility First [14]. By using internet, ocean data can be broadcasted in different applications like scientific applications, military applications, industrial applications and civilian applications.

This paper is organized as follows. Section II explains Internet of Underwater Things Architecture using Mobility First. Section III shows internal architecture of Underwater Thing. Section IV explains Assigning GUIDs. Section V gives the details of Delay and Disruption Tolerant Network. Section VI explains applications of IoUT. Section XII deals with the GUID results. In the last Section, we conclude our paper and outline future works.

\section{Architecture of IoUT Using Mobility First •}

The proposed IoUT architecture is shown in Figune 1y it has three jayers IoUT layer, DTN Communication layer and IoT layer.

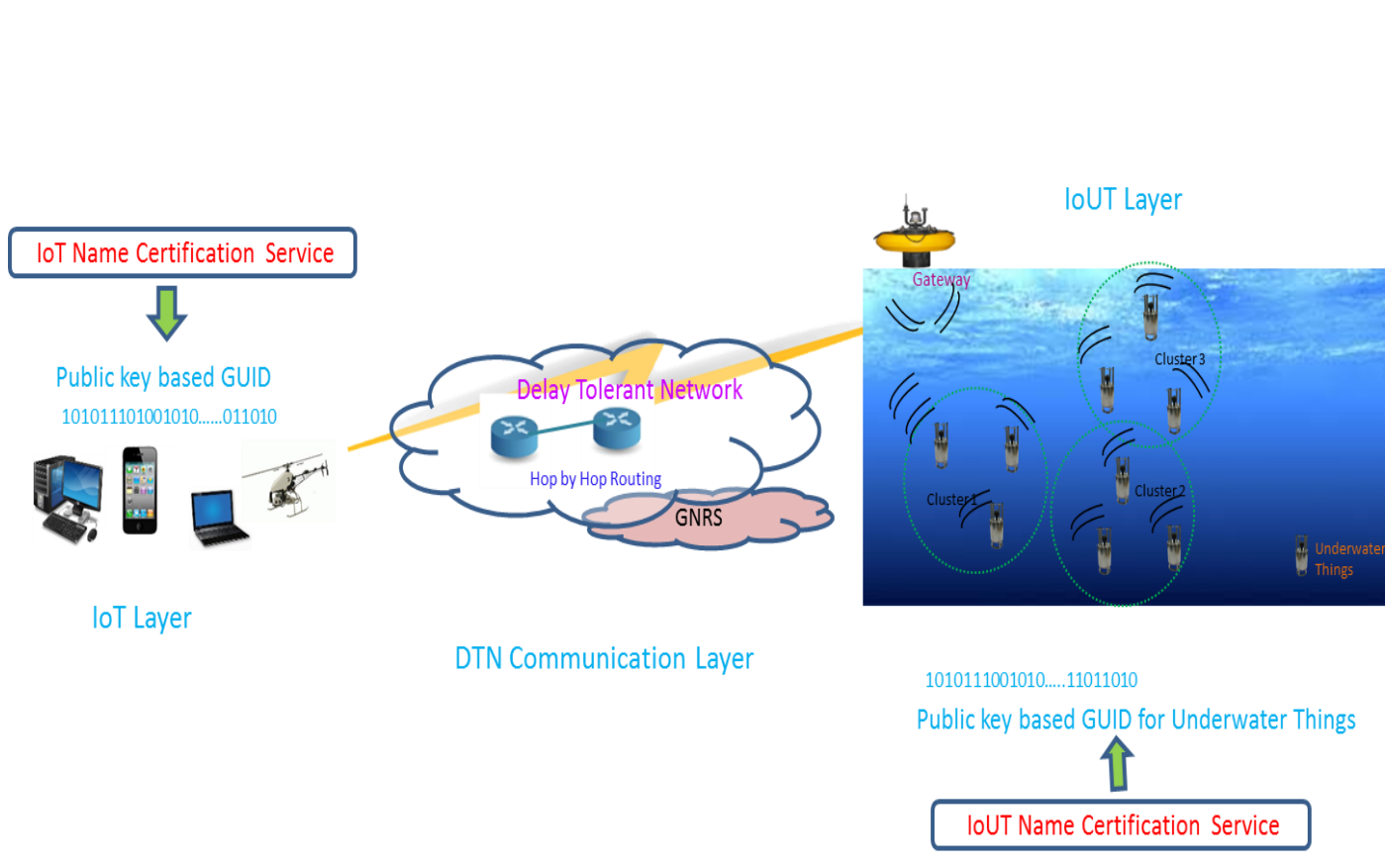

Figure 1. Architecture of loUT using Mobility First

IoUT layer gathers the information from underwater and communicate to IoT layer with the help of DTN communication layer. Underwater things query the GNRS for IoT mapping with the help of gateway. GNRS provides IoT network information to the underwater things. Communication between IoT layer and IoUT layer happens with the help of DTN nodes. DTN nodes store and forward mechanism is used between the DTN nodes to pass the information. Reliable hop by hop transmission is used in DTN communication layer.

\subsection{IoUT Layer}

IoUT layer is associated to underwater environment and collects the information related to the underwater environment. Underwater things can gather the 
information like $\mathrm{pH}, \mathrm{CO} 2$, and current temperature etc., with the help of different kind sensors. Underwater thing equipped with sensors, which allows the inspection of underwater properties and measuring of technical information in collaborative monitoring applications. These applications are disaster anticipation, offshore exploration, and strategic inspection so on [9]. IoUT Name Certification Service (NCS) assigns the public key based GUIDs to underwater things.

\subsection{DTN Communication Layer}

Delay and Disruption Tolerant Network (DTN) is used to establish communication between heterogeneous networks. In this paper DTN is used for establishing communication between underwater acoustic sensor networks and RF (Radio Frequency) communication [18]. In this layer storage aware DTN routing enables the access of the data from underwater and transmits the required information to the base station center hop by hop transmission. DTN routing protocols are considered to support network storage whep essentiah to overwhelmed connection quality variations and disconnections [171. GNRS is assed to mapping between the GUIDs to corresponding NAs. Mobinty First intra domain routing protocols are used to support store and forward meehanism when fluctuations and disconnections occur [10].

\subsection{IoT Layer}

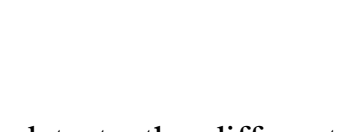

This layer provides the underwater data to the different things like mobile devices, PCs, laptops and air vehicles. IoT Name Certification Service (NCS) assigns public key based GUIDs to the things.

\section{Underwater Thing}

In IoUT layer under thater things are used. Underwater thing is assembled with various components like transducers, sensor interfaces, sensors, Medium Access Control (MAC) board, underwater modem, battery for power supply etc. Underwater thing is designed with different kind'of sensors. Each underwater thing is equipped different sensors like $\mathrm{pH}$, turbidity and temperature sensors and related sensor interfaces. Based on the sensor type underwater thing can send appropriate data. Underwater thing is protected with underwater proofing case as shown in Figure 2. Underwater thing is pulled down into underwater by the sea anchor with the help of chain. Battery is placed in the underwater thing, to supply electric power to underwater modem, MAC board and sensors. Transducer converts the electric signal corresponding to the sending data to the acoustic signal. Transducer is one of the main internal components of the underwater thing. Figure 2 show the internal architecture of underwater thing.

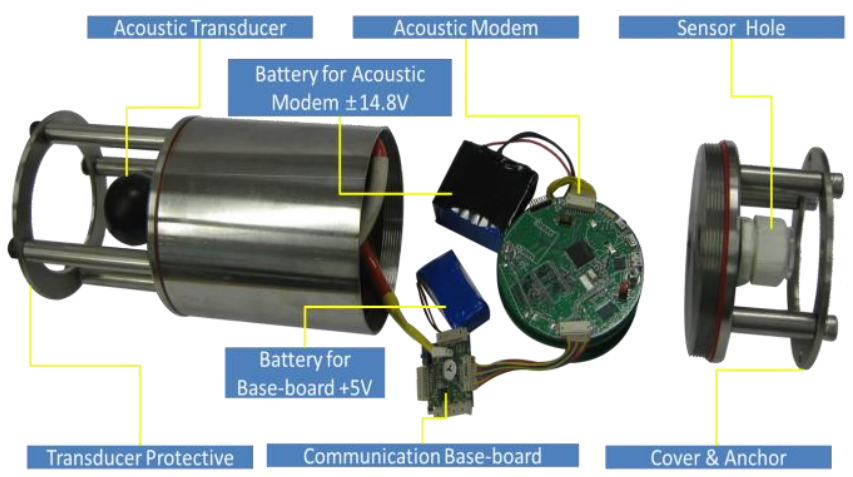

Figure 2. Internal Architecture of Underwater Thing 
In this section we explain the internal architecture of underwater thing available for the IoUT. Underwater thing can have three functionalities.

\subsection{Sensing}

For sensing sensor and sensor interfaces have been used together. Sensors are used to sense the $\mathrm{pH}, \mathrm{CO} 2$, turbidity, etc. Sensor interface is used to make interfacing among sensors and main board.

\subsection{Processing}

Microcontroller contains a processor core, memory, and programmable input/output peripherals. In our experiments, we used Cortex-M3 MCU (Micro-Controller Unit). Microcontroller process the sensing data. Cortex-M3 is used as MCU to drive the underwater acoustic modem.

\subsection{Communication}

Underwater acoustic modem, MAC Board and transaucer are used in underwater thing communication. Acoustic modem directly connects to the MAC Board for underwater communication. Underwater acoustic modem contains of thee cireular boards. They are an analog transmission layer, a digital layer and an analog reception layer. The underwater acoustic modem is equipped gith STM32 as a main processor, Serial Peripheral Interface (SPI) and Universal Asynchronous Receiver Transmitter (UART) communication ports for interlocking with upper layer or other device. Transducer converts electrical signal into acoustic signal and vice versa. This transducer conducts 70 $\mathrm{KHz}$ frequency and half-duplex communication with underwater modem.

\section{Assigning GUIDs}

Internet of Underwater Things (goUT) faces two major challenges. One is the universal identity of underwater things and other is standardizations of the data format for underwater things. Enabling the IoU requires a global identification system for things to be distinguished from trillions of internet things and underwater things and to make them globally accessible from the internet. The current Internet architecture uses an IP address as both ID and locafor ror identification of thing. In the internet physical objects are identified at product level through attached tags and network objects are directly identified at application level. In the current Internet, both physical and network objects cannot be addressed or identified directly by the core network which leaves the IoUT just another type of applications.

The (IP protocol stacks for underwater environment is explained [16]. One approach is to extend IP addressing from computers to underwater things, i.e., assigning all things an IPv6,address, which has a huge addressing space. IPv6 defines the lowest 6 to 8 bytes of addressing space as device ID, inherent from the 6 bytes of Medium Access Control (MAC) addresses. Although 6 bytes are long enough for identifying all physical objects connecting to the Internet, there are a few fundamental issues with directly using IPv6 addresses as object identities. Firstly, it does not support mobility. The devices' dynamic connectivity or lost connectivity and multi-homing cannot be handled efficiently in IPv6, and IP tunneling may be necessary between different operators. Secondly, it is inherently insecure at the network layer; neither the source nor the destination can prove their authorities through their identities. Most sensors and tags may not be able to run an IP stack directly due to their cost and energy constraints. Therefore, there is no need to stick on IPv6 for assigning network identities to underwater things. For these reasons the concept of GUIDs is introduced for IoUT. 


\subsection{GUID (Global Unique Identification System)}

Global Unique Identification (GUID) technique is the separation of naming and addressing. Every Thing is assigned with a GUID, which is independent of its addressing scheme and locations. Fig. 3 shows the internal architecture of GUID.

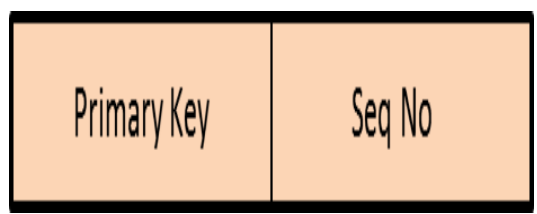

Figure 3. GUID Structure

The first principle of IoT and IoUT is the name and address separation, giving every network object a GUID independent of its network address. In legacy systems GUID Address is defined by 128 bits. Realization of GUID with 128 bits has implementation issues in under water. RF communication does not work well in the underwater because of attenuation. Instead of RF communication, acoustic communication is used in under water. But acoustic signals have low bandwiath and long propagation delays [1]. Underwater things are usually powered by batteries, which are difficult to recharging and replacing in underwater environment [8]. So implementing GUID in underwater environment is one of the challenging issues. Hashing is the application of a function to the key value that results in mapping the range of possible key values into a smaller range of relative addresses. GUID structure can be formed als owner public key and sequence number as shown in Figure 3. An øyner may use one key pair for multiple underwater things he owns with sequence numbers. NCS is an owner selected service to publish a GUID for underwater things he/she owns, It maps a human readable semantic string, for example, "temperature of underwater" toa GUID.

\subsection{Global Name Resolution Service}

Global Name Resolution Serviced(GNRS) provides the mapping between GUIDs and the corresponding NAs [1 1 GNRS [4] is similar to today's DNS (Domain Name Server) service which maps a Unifom Resource Locator (URL) to an IP address.

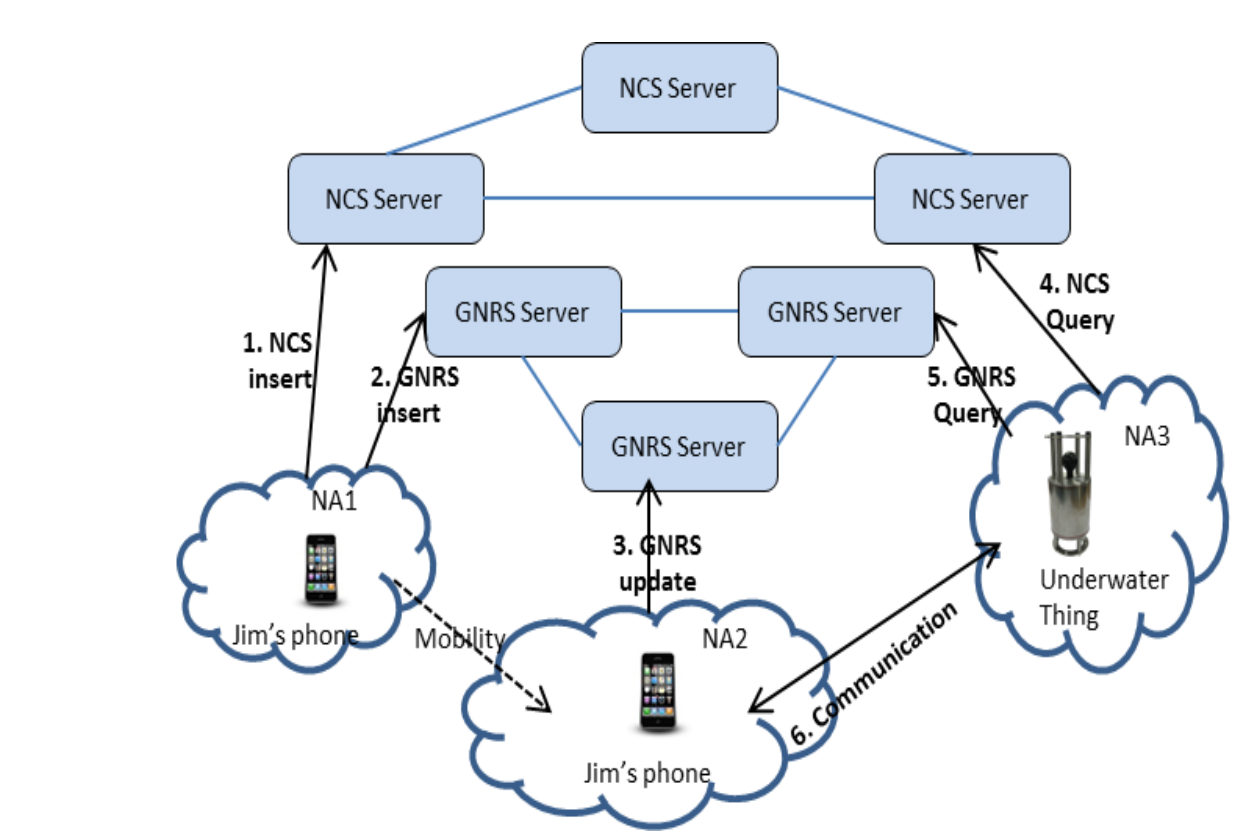

Figure 4. Global Name Resolution Service 
There are three types of events in GNRS: insert, update and query operations. These operations are shown in Figure 4. When Jim's phone moves to another network, it sends an update message to the GNRS to report the new mapping. If another underwater thing wants to communicate with Jim's phone, it sends a query message to the GNRS asking for Jim's phone network address before their communication, and then GNRS will return Jim's phone mapping to underwater thing.

4.2.1. GNRS Query Operation: In the GNRS query operation, assume Thing1 is in network NA1 and wants to query Thing2. GNRS query operation is shown in the Fig. 5. Thing 1 first sends out the query request message to its border gateway router. The border gateway router forwards the query request message to GNRS for Thing2 mapping. GNRS returns the Thing2 mapping to Thing1's border gateway router, who wrill then reply to Thing1's query with the mapping information. Using the mapping information Thing1 communicate with Thing2.

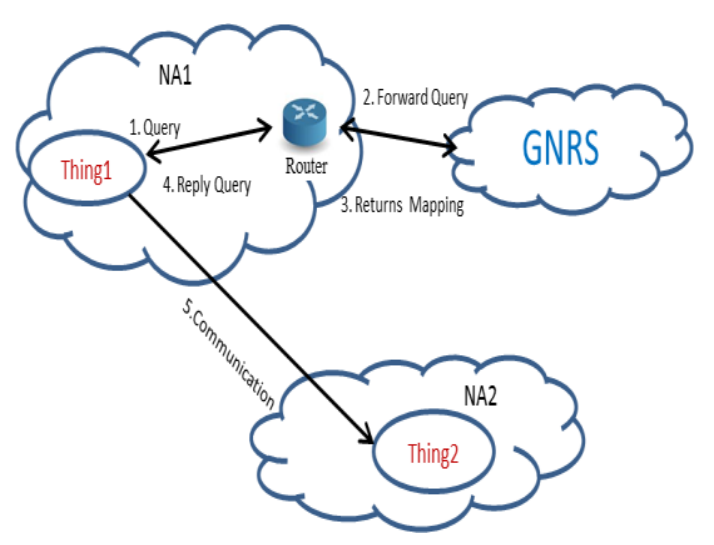

\section{Figure 5.) GNRS Query Operation}

4.2.2. GNRS Update Operation In the GNRS update operation, suppose Thing1 belongs to network NA1 and needs to update its mapping (GUID, NA2). Thing1 sends an update message to the border gateway router. The border gateway router sends the update query to GNRS as shown in Figure 6.

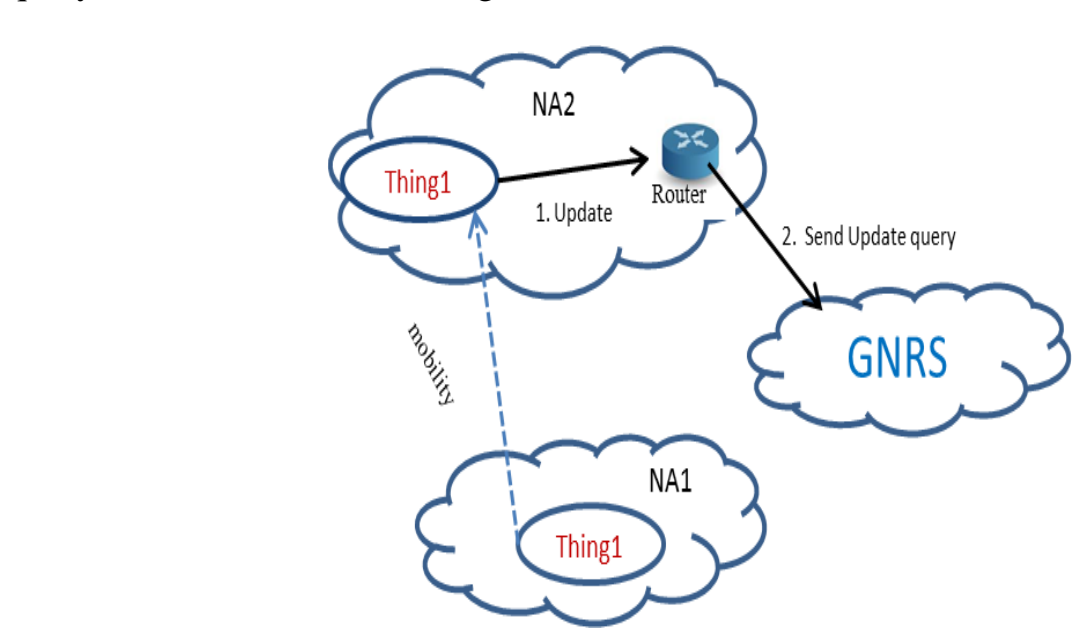

Figure 6. GNRS Update Operation

\subsection{Name Certification Service}

The NCS provides translation between human readable name and GUID. It acts as a certificate authority in distributing the public key based GUIDs. 


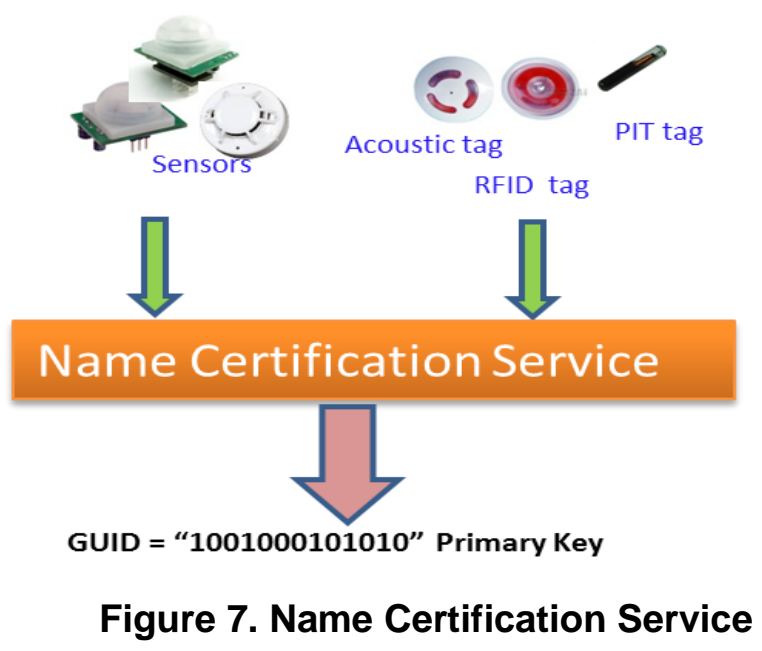

Figure 7 shows example assignment of GUIDs to sensors, acoustic tags, PIT tags and RFID tags. NCS provides a facility to generate automatic user described public keys to use as GUIDs.

\subsection{Naming Convention and Mapping}

GUID technique is the separation of naming and addressing. Every network object is assigned with GUID, which is independent of its addressing scheme and locations. Naming Certification Service (NCS) assigns the CUHDs to different underwater things. Name convention and mapping is shown in Figure 8. Global Name Resolution Service (GNRS) provides the mapping between GUIDs and the corresponding NAs.

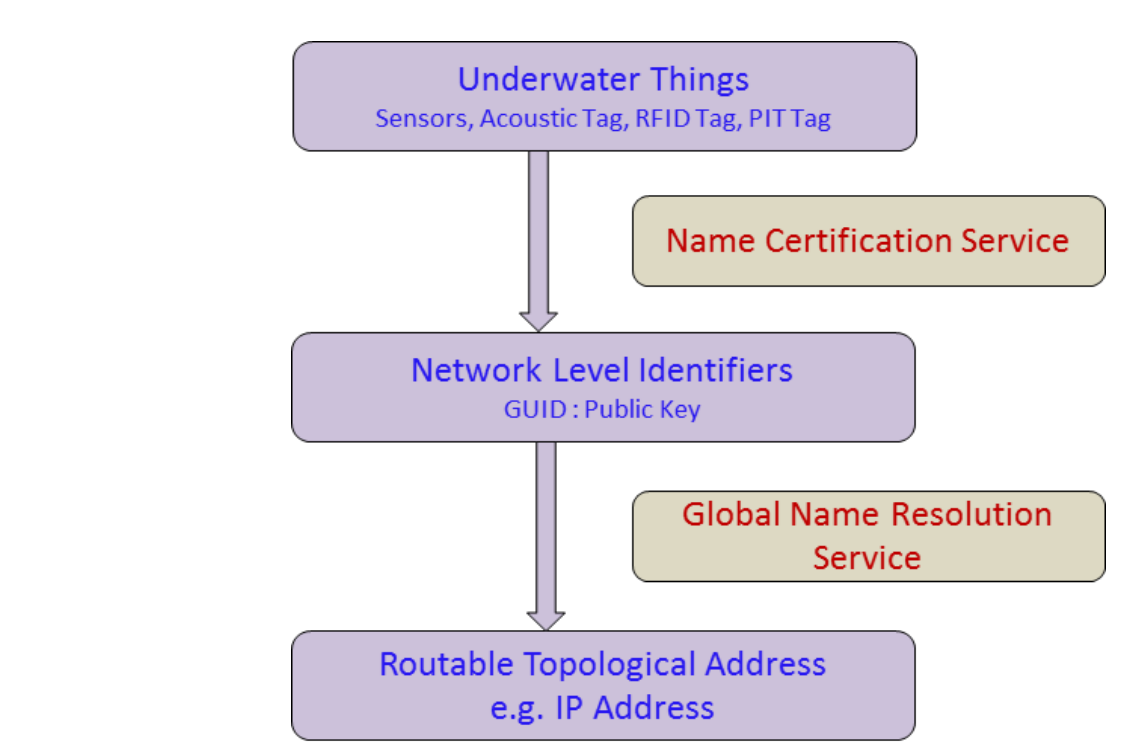

Figure 8. Naming Convention and Mapping

\subsection{Steps in Message Delivery between Two Things}

As shown in Figure 9, the user registers his devices such as mobile phone and laptop to the NCS and NCS assigns GUIDs to the user devices, i.e., IoT things. 


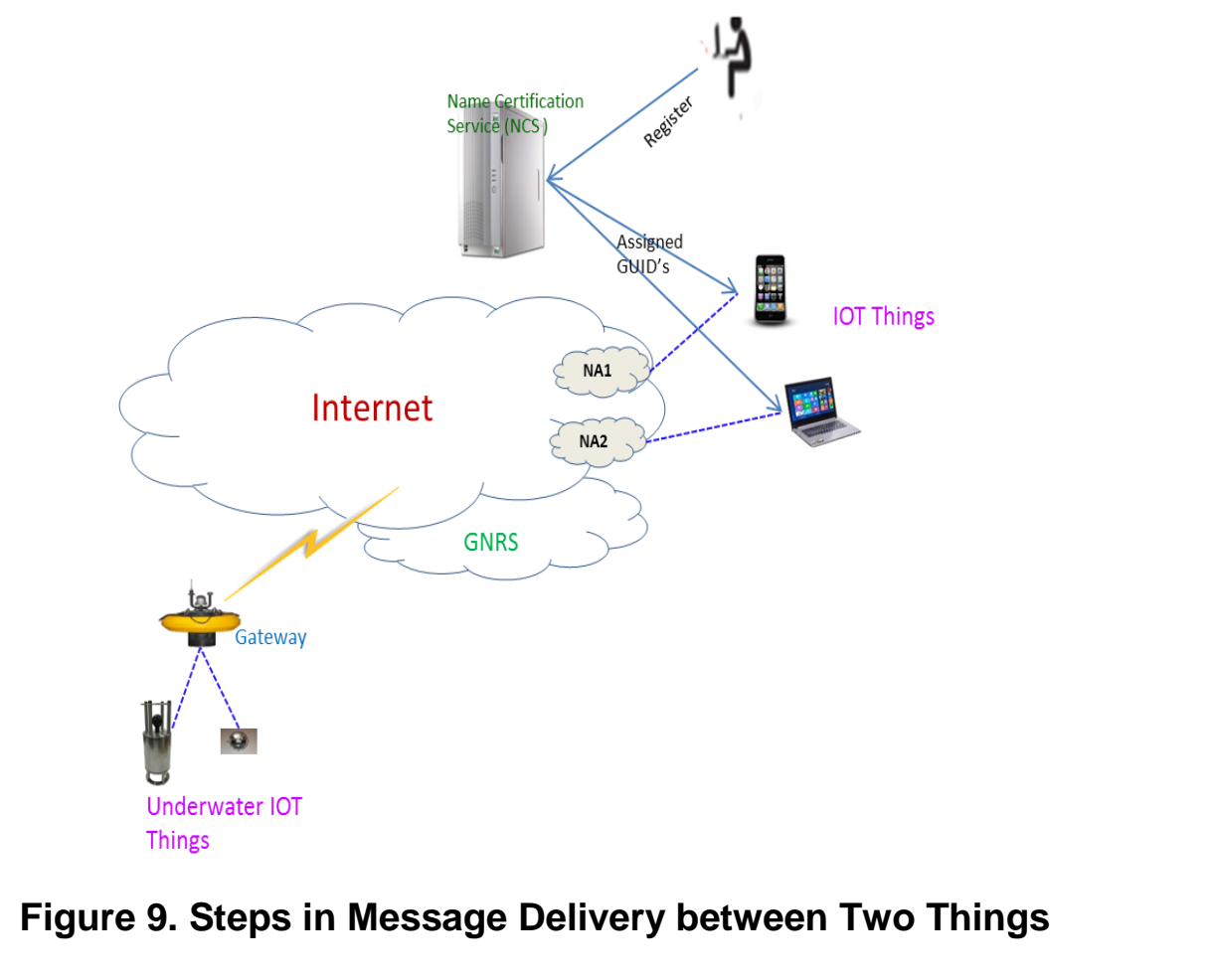

An Underwater thing wishing to send a message to all of IoT Things like mobile or laptop will obtain the corresponding GUID from either the NCS or the end user. The underwater thing sends GNRS query to its gateway for IoT things network addresses corresponding to the current ponts of attachment of user devices, in this case NA1 and NA2. GNRS provides exact location of - Jor things to under water IoUT things. So they both can communicate successfully. If a delivery failure occurs due to disconnection or mobility, the packet is stored inside the network and the GNRS is periodically queried for a rebinding of the GUD with NAs.

\section{Delay and Disruption Tolerant Network}

Delay and Disraption Tolerant Network (DTN) is useful for establishing communication among heterogeneous networks. In this paper we used DTN to establish communication between underwater acoustic sensor networks and RF communication network [13].1

Store and forward mechanism is used in the DTN for efficient communication among heterogeneous networks. Underwater acoustic communication is influenced bx path loss, noise, attenuation and propagation delay. Due to these reasons DTN [2] is very useful communication technique in underwater. Figure 10 shows the example of DTN which is used in IoUT. In our architecture IoT layer uses the RF communication techniques and IoUT layer uses the acoustic communication mechanisms. Underwater acoustic communication [7] is influenced by ecological parameters such as temperature, density and conductivity. Also bandwidth is low in underwater acoustic communication, whereas bandwidth is high in RF communication.

DTNs overcome the problems associated with intermittent connectivity, long or variable delay, asymmetric data rates, and high error rates by using store-andforward message switching. Whole messages (entire blocks of application program user data) of such messages are forwarded from a storage place on one node to a storage place on another node, along a path that eventually reaches the destination. The nodes of DTN need storage, because communication link to the next node may 
not be available for a long time. If one of the communicating node is sending data much faster than the other node then DTN node must maintain storage. If transmission error occurs the data must be resent. So DTN needs storage for above reasons.

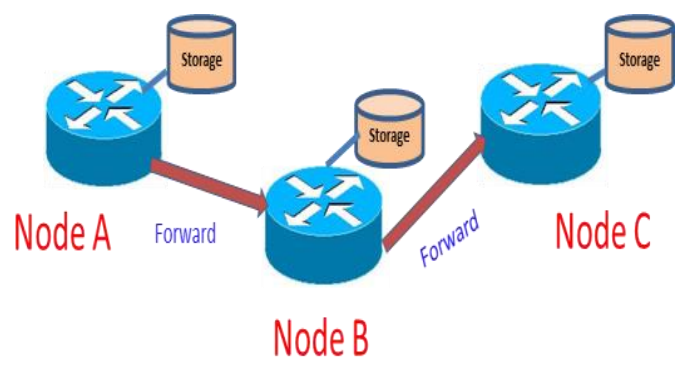

Figure 10. Example of DTN in loUT

In Figure 10 DTN nodes are shown to transfer data hop by hop. Node A forwards the data to the node $\mathrm{B}$, once data reaches successfylly node $\mathrm{A}$ removes the data from its storage. If any failure occurs in data transmission node A resends the data. In the same way data is transmitted to node $\mathbf{C}$ from node $B$.

\section{Applications of IoUT}

Applications of IoUT fall into four categories?

\subsection{Scientific Applications}

In the scientific applications [12] Underwater thing observes the ocean characteristics like oxygen levels, temperature, salinity, bacterial and other pollutant content, dissolved matter, etc., with the help of different sensors to counting or imaging animal life of micro-organisms, fish or mammals.

6.1.1. Environmental Monitoring - Disaster Prevention: Tsunami is a series of long water waves caused by underwater earthquakes. Underwater things can measure the seismigactivity from remote locations and provide Tsunami warnings to coastal areas, on tudy the effects of submarine earthquakes.

\subsection{Industrial Applications}

Undervater things can be widely used in Industrial applications [3]. These Industrial applications monitor and control commercial activities, such as underyater equipment related to oil or mineral extraction, underwater pipelines or commercial fisheries. Industrial applications involve in control and actuation components.

6.2.1. Fish Farming: Fish agricultural is the wildest growing food manufacturing segment in the globe. Computer controlled and automatic remote monitoring intensive cultivation is the upcoming development in fish farming. Water quality plays a significant role in the fish farming. Proper control of the water quality to preserve the concentration of the water atmosphere constraints in the optimum range can improve the fish development rate, affect nutritional consumption and decrease the amount of large scale fish illnesses. Without collecting information of physical and biochemical constraints of water quality together with the associated environmental aspects, it is practically difficult to accomplish the appropriate water quality control at right place in right time. In traditional methods, samples of water 
send to a chemical laboratory to examine the hazardous ingredients. It is time consuming process. Instead of traditional methods, underwater things can be used to check the level of water quality.

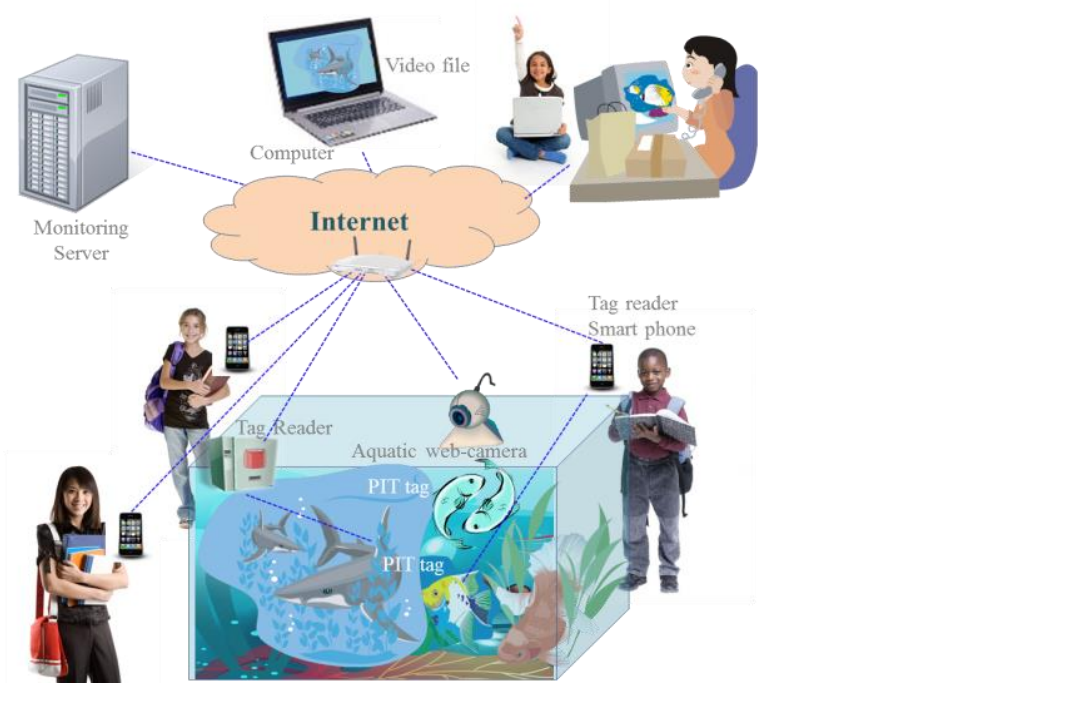

Figure 11. Fish Farming

Fish Farming scenario is shownefiffígure 11. Numerous underwater things can constantly read certain parameters, which designate the water excellence level such as temperature, $\mathrm{pH}$, salinity, dissolved oxygen, turbidity, etc. Fish information monitoring is planned to be carried out from a remote location. So the information from underwater things will, be sentwirelessly to the base station.

6.2.2. Pipeline Monitoring: Pipelines are an essential part of shipping the hydrocarbons to downstream-processes in underwater oil and gas construction fields. Comflications may arise in pipelines because of deterioration, mechanical failure and mud formation due to hydrocarbon biochemical processes. These kinds of difficulties lead to manufacture, revenue defeat in addition to high maintenance expenditures. Additionally, today's ecological concerns govern that hydrocarbon leaks into the ocean be avoided to prevent environmental disasters. Pipeline monitoring scenorio is shown in Figure 12. Decreasing these difficulties by anticipating and appropriate action is of vital attentiveness to the productiveness. Continuous pipeline monitoring delivers the necessary information to make the accurate decisions.

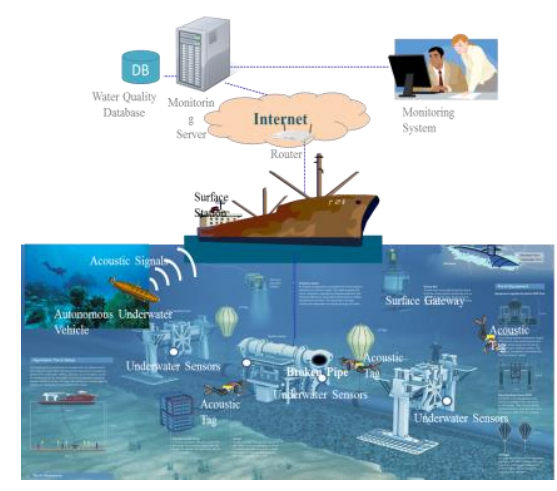

Figure 12. Monitoring of Pipelines 
Underwater things and sensors are located along the pipeline to gather data that can be transported to the surface.

6.2.3. Environmental Monitoring - Chemical and Biological Changes: In Oceanographic underwater things are used to record and capture a multitude of oceanographic variables such as ocean currents, salinity, temperature, pressure, and dissolved oxygen with the help of sensors. Underwater things can be used to detail the chemical slurry of antibiotics, estrogen-type hormones and insecticides to monitor streams, rivers, lakes and ocean bays. Monitoring of ocean currents and winds, improved weather forecast, detecting climate change, understanding and predicting the effect of human activities on marine ecosystems, biological monitoring such as tracking of fishes or micro-organisms, are other possible applications, where underwater thing is useful.

6.2.4. Undersea Examination: Underwater things can help to detect underwater oilfields or reservoirs, determine routes for laying undersea cables, and assist in exploration for valuable minerals.

\subsection{Military Applications}

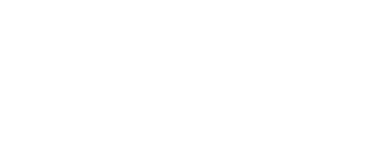

Military and home land security applications [3] inyolve/securing or monitoring port facilities or ships in foreign barbor's, deminifg, and communicating with submarines and divers. Underwater things can be used in military applications in the sea.

6.3.1. Distributed Strategic Surveillance: $y$ AUVs and underwater things can collaboratively monitor areas for surveillance, reconnaissance, targeting and intrusion detection systeres.) For example, an underwater thing which is designed for a tactical surveillancersystem that is able to detect and classify submarines, Small Delivery Vehicles (SDVs) and givers based on the sensed data from mechanical, radiation, magnefic and acoustic micro sensors which are fixed in the underwater thing. Underwater things can reach a higher accuracy, and enable detection and classification of low sighature targets by also combining measures from different types of sensors fixed the underwater thing. Figure 13 shows the strategic surveillance scenario

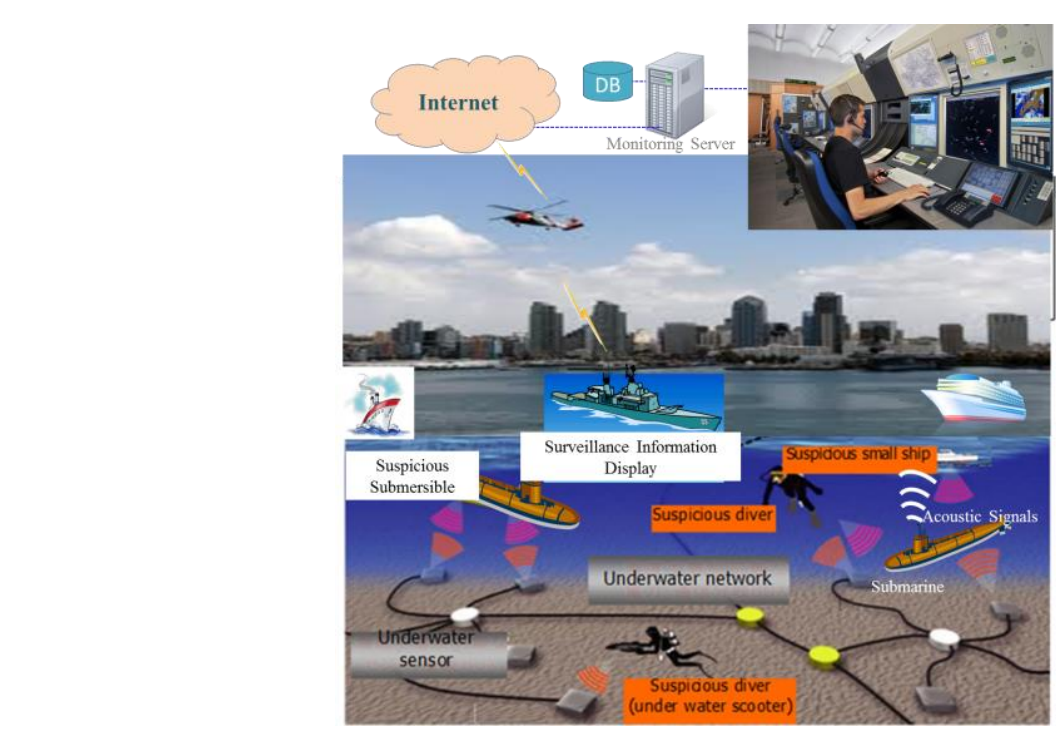

Figure 13. Strategic Surveillance 
Underwater things together with underwater and surface vehicles can contribute to protect harbors. Stationary platforms that contain buoys and sea-mounted equipment are also included. A large variety of sensors can be mounted on the vehicles. Another certain number of underwater things are deployed to cover a large volume of water. The sensed data is sent to the surface station (sink), which floats on the water surface and uses long distance radio communication to send this data to an onshore station directly. Further analysis of the data is performed at the onshore station. These underwater things can search for intruder submarines and send alert messages to AUVs in case of detection.

\subsection{Civilian Applications}

6.4.1. Assisted Navigation: Underwater things can be used to identify hazards on the seabed, locate dangerous rocks or shoals in shallow waters, mooring positions, submerged wrecks, and to perform bathymetry profiling with the help of'sensors. Assisted navigation scenario is shown in Figure 14.

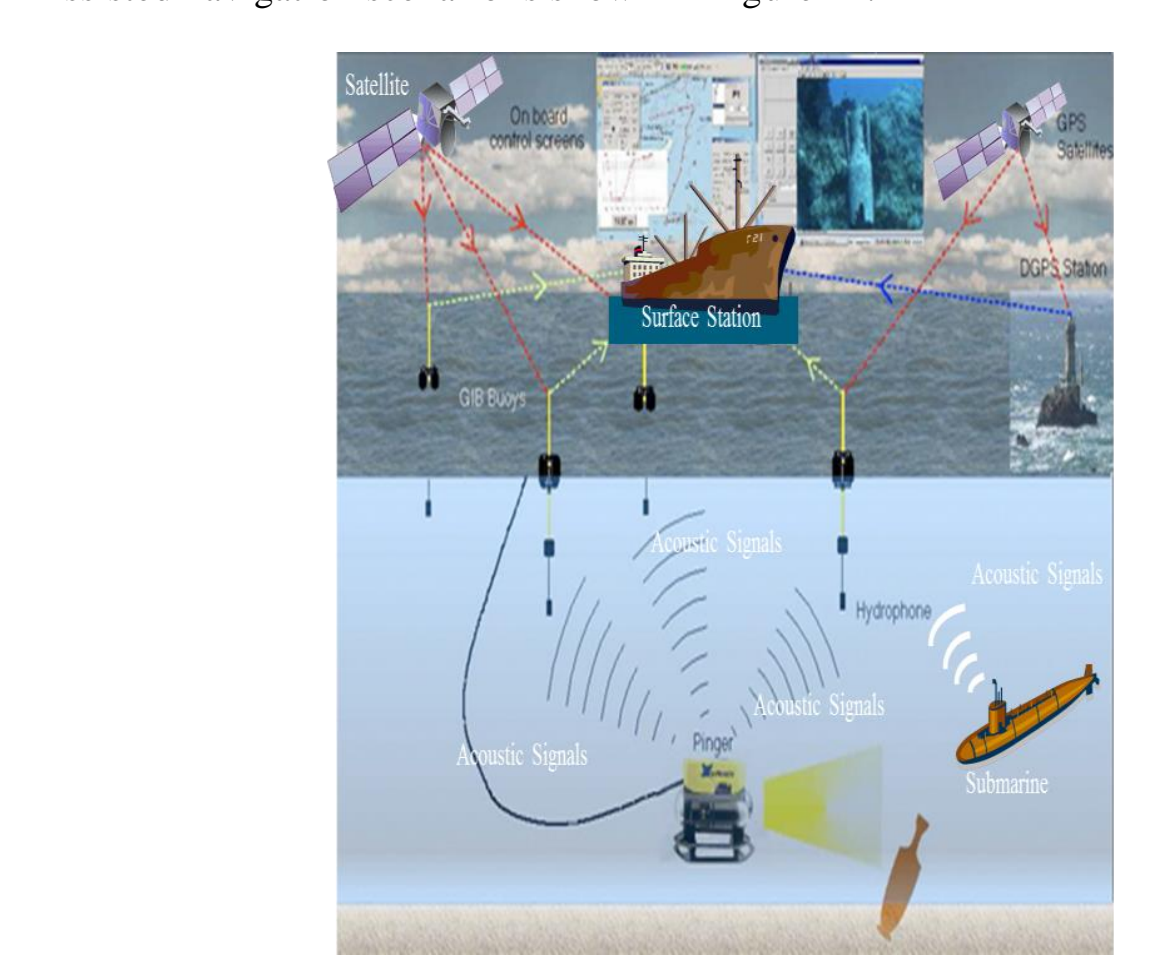

\section{Figure 14. Assisted Navigation}

\section{Results and Discussion}

The number of queries for any GUID depends on its popularity amongst Internet and underwater hosts. In order to capture the effects of the wide variations in the host popularity, Mandelbrot-Zipf distribution can be used to model the varying host popularity. Mandelbrot-Zipf distribution is the general form of Zipf-like distributions. The rank distribution of the websites follows the Zipf law. The Mandelbrot-Zipf distribution [2] defines the probability of accessing an object at rank $\mathrm{r}$ out of $\mathrm{N}$ available objects as:

$$
\mathrm{P}(\mathrm{r})=\mathrm{H} /(\mathrm{r}+\mathrm{q})^{\alpha}
$$

Where $\mathrm{H}=1 / \sum \mathrm{Nr}=11 /(\mathrm{r}+\mathrm{q})$ awith a determining the skewness ' $\alpha$ ' and plateau factor ' $\mathrm{q}$ ' affecting the "flatness" of the peak. We use a value of $\alpha=1.02, \mathrm{q}=100$ 
following arguments [15]. The probability of accessing GUIDs is shown in the Table 1.

Table 1. Probability of Accessing a GUID

\begin{tabular}{|c|c|c|}
\hline $\begin{array}{c}\text { No of } \\
\text { GUIDs }\end{array}$ & $\begin{array}{l}\text { Highest } \\
\text { Probability }\end{array}$ & $\begin{array}{l}\text { Lowest } \\
\text { Probability }\end{array}$ \\
\hline 10 & 0.1091 & 0.1000 \\
\hline 20 & 0.0596 & 0.0500 \\
\hline 30 & 0.0250 & 0.0193 \\
\hline 40 & 0.0146 & 0.0104 \\
\hline 50 & 0.0098 & 0.0065 \\
\hline 60 & 0.0072 & 0.0045 \\
\hline 70 & 0.0055 & 0.0033 \\
\hline 80 & 0.0044 & 0.0025 \\
\hline 90 & 0.0037 & 0.0019 \\
\hline 100 & 0.0031 & 0.0015 \\
\hline
\end{tabular}

Table 1 shows the number of GUIDs, probability of accessing popular GUID and probability of accessing least popular GUID. The graphis drawn lsing this table.

Figure 15 shows GUID probabilities for $\alpha=1.02$ and $\mathrm{q}=100$. One curve drawn based on the probability of using popular GUIDand another curve drawn based on the probability of using least popular GUID for number of GUIDs.

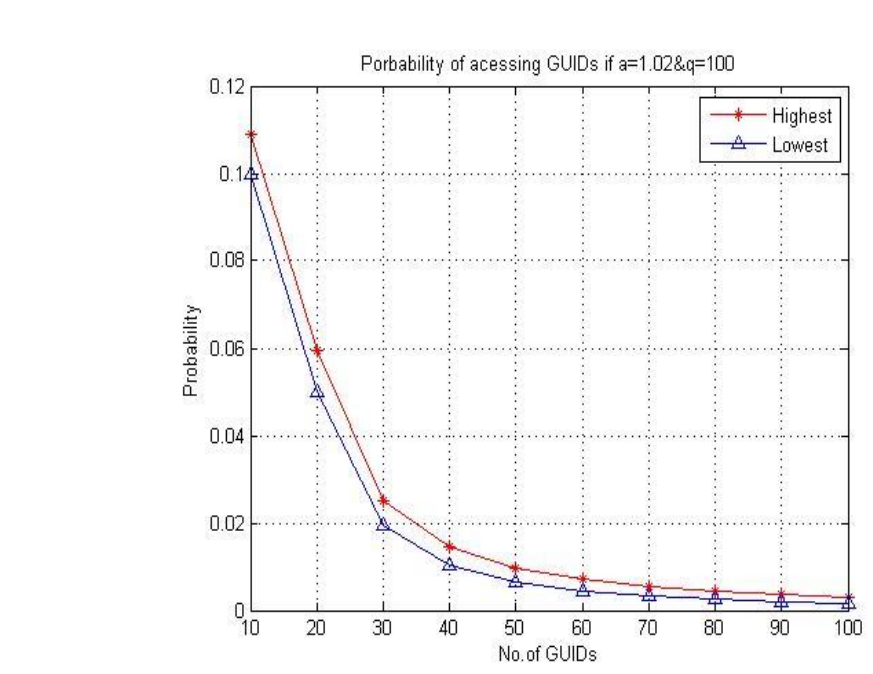

Figure 155. GUID Probabilities for $\alpha=1.02$ and $q=100$

$\mathrm{X}$-axis Shows the number of GUIDs on the scale of 10 and $\mathrm{Y}$-axis shows the probability. The higher value of $q$ means the objects are requested less often. A sinal yalue of ' $\alpha$ ' and a large value of q resulting in popularity being spread out among objects more evenly [15]. That is approaching a uniform distribution.

In Figure 16, GUID probabilities are calculated using $\alpha=0.65$ and $q=5$. Here highest probability of using a GUID among 10 GUIDs is 0.1814 and least probability of using a GUID among 10 GUIDs is 0.1000 . In the same way probabilities are calculated for 20, 30, 40, 50, 60, 70, 80, 90 and 100 GUIDs. Highest probability of using a GUID among 100 GUIDs is 0.0086 and least probability of using a GUID among 100 GUIDs is 0.0018 . 


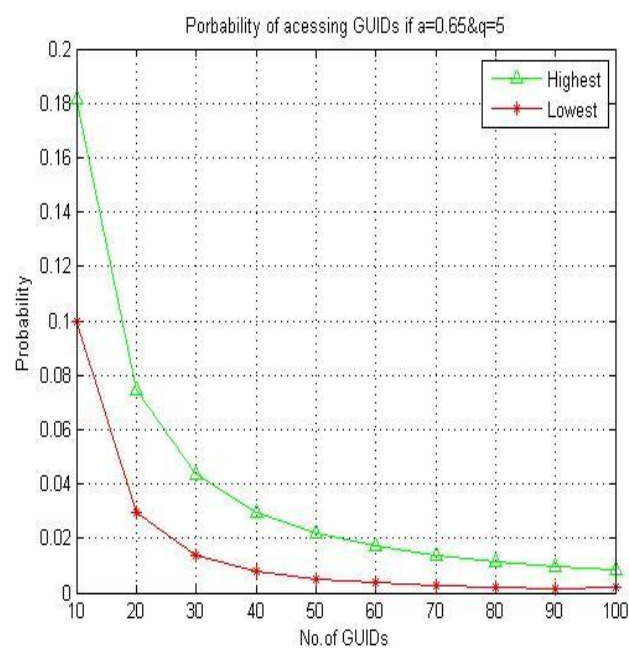

Figure 166. GUID Probabilities for $a=0.65$ and $q=5$

In Figure 17, GUID probabilities are calcalated using $\alpha=0.55$ and $q=25$. Here highest probability of using a GUID among 10 GUDS is 0.1178 and least probability of using a GUID among 10 GUIDs is 0.0999. In the same manner probabilities are calculated for 20, 30, 40, 50, 60, 70, 80, 90 and 100 GUIDs. Highest probability of using a GHD among 100 GUIDs is 0.0032 and least probability of using a GUID among 100 GUIDs is 0.0015 .

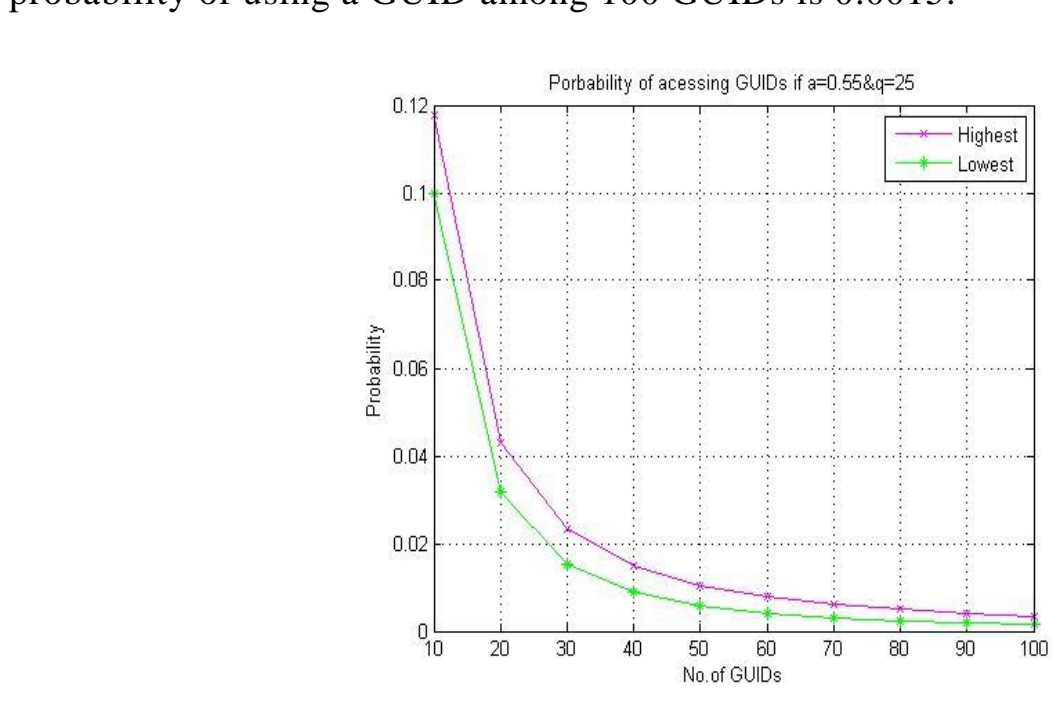

Figure 17. GUID Probabilities for $\alpha=0.55$ and $q=25$

In Figure 18, GUID probabilities are calculated using $\alpha=0.60$ and $\mathrm{q}=40$. Here highest probability of using a GUID among 10 GUIDs is 0.1126 and least probability of using a GUID among 10 GUIDs is 0.0999 . With the same approach probabilities are calculated for 20, 30, 40, 50, 60, 70, 80, 90 and 100 GUIDs. Highest probability of using a GUID among 100 GUIDs is 0.0029 and least probability of using a GUID among 100 GUIDs is 0.0017 . 


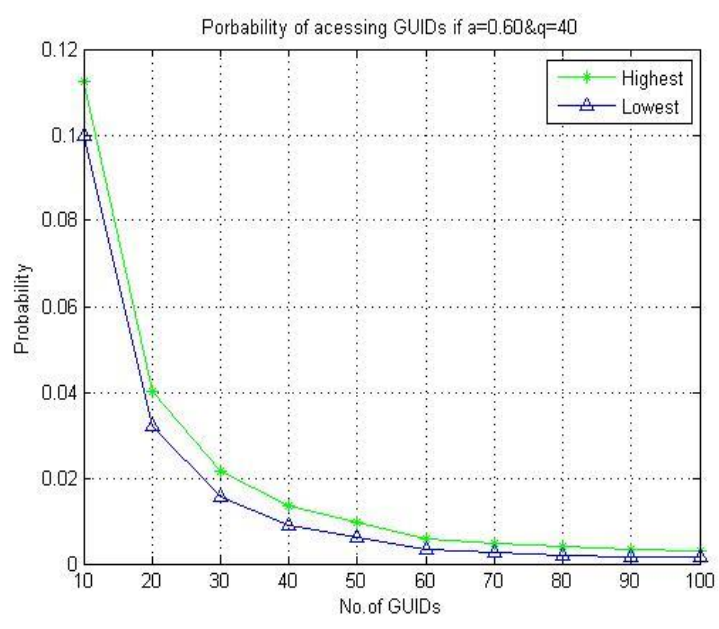

Figure 18. GUID Probabilities for $\alpha=0.60$ and $q=40$

In Figure 19, least used GUID probabilities are calculated with)different $\alpha$ and $\mathrm{q}$ values. Graph is drawn based on the probability of using least popular GUID for number of GUIDs. X-axis shows the number of GUIDs on the scale of 10 and Yaxis shows the probability. Four curves afe drawn based on the different $q$ values like 5, 25, 40 and 100 .

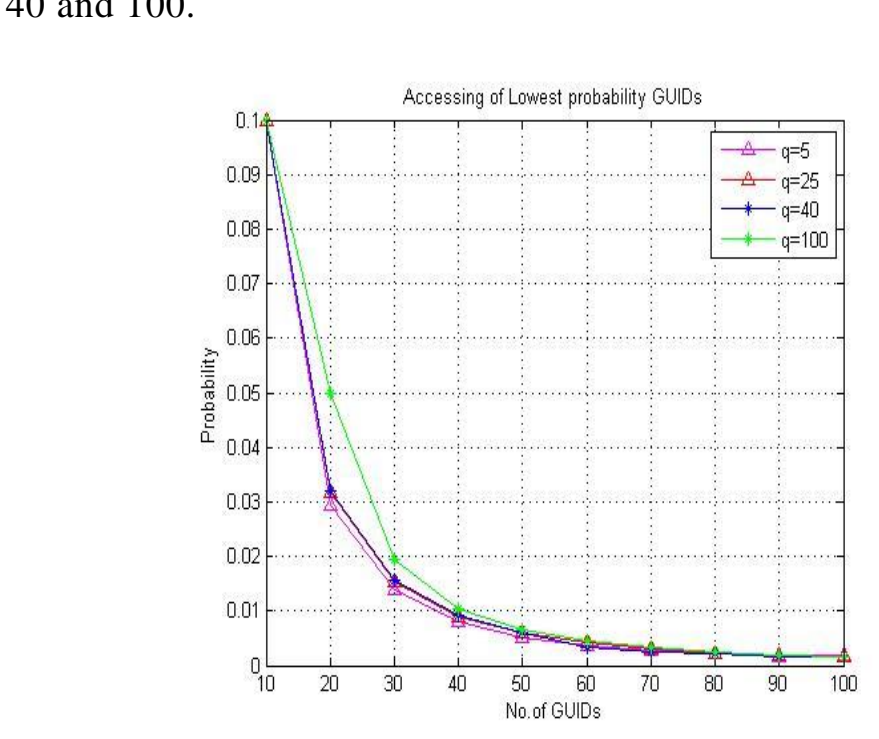

Figure 199. Least Used GUID Probabilities

In Figure 20, most used GUID probabilities are calculated with different $\alpha$ and $q$ values. Graph is drawn based on the probability of using most popular GUID for number of GUIDs. 


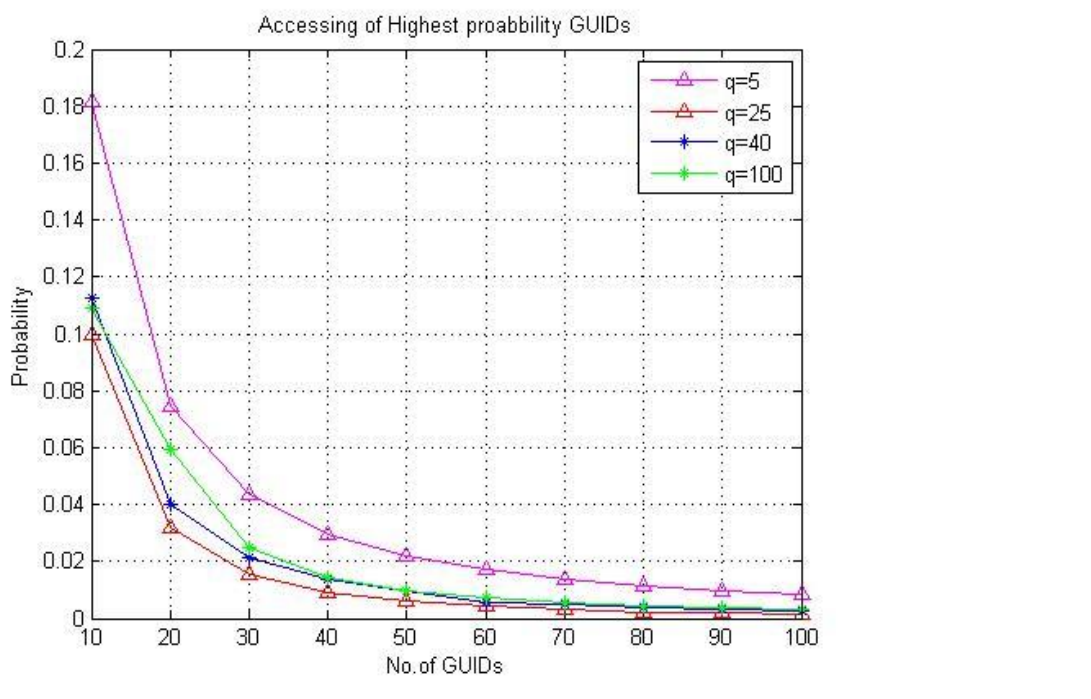

Figure 20. Most Used GUID Rrobabilities

$\mathrm{X}$-axis shows the number of GUIDs on the scale of 10 and $\mathrm{Y}$-axis shows the probability. Four curves are drawn based onthe different q yalues like 5, 25, 40 and 100 .

\section{Conclusion}

In this paper GUID for Mobility First architecture is used to support the IoUT. In this architecture we explained IoUT layer with help of underwater things, DTN communication layer with GNRS and LoT layer with NCS. In underwater environment underwater things are used to sense different kinds of information like $\mathrm{pH}$, Temperature and Turbidity. Internal architecture of underwater thing helps to sense, process and communication. Assignment of GUIDs is done by NCS in Mobility First Architecture. GUID contains primary key and sequence number. Functioning of the GNRS is explained using query and update operations. In this paper naming convention and mapping is explained with the help OSNCS. Detailed steps are given to deliver the message between two things. Functionality of DTN is explained with store and forward mechanism. IoUT is useful in scientific, military, industrial and civilian applications. Environmental monitoring and disáster prevention comes under scientific applications. Fish farming, pipeline monitoring and undersea explorations comes under industrial applications. Strategic surveillance is a kind of military application. Assisted navigation is a civilian application. Finally results are shown with least used GUID probabilities and most used GUVD probabilities.

\section{Acknowledgement}

The work is a part of the results of the research "Development of the wide-band underwater mobile communication systems" supported by Ministry of Oceans and Fisheries, Korea.

\section{References}

[1] A. Caiti, K. Grythe, J. M. Hovem, S. M. Jesus, Lie, A. Munafo, T. A. Reinen, and F. Zabel, "J. Ocean Engineering", Linking Acoustic Communications and Network Performance: Integration and Experimentation of an Underwater Acoustic Network, vol. 38, (2013).

[2] Z. Silagadze, "J. Complex Systems. Citations and the Zipf-Mandelbrot's law", vol. 11, no. 487, (1997). 
[3] M. Carmen and Domingo, "J. Network and Computer Applications", An overview of the internet of underwater things, vol. 35 , no. $1879,(\mathbf{2 0 1 2})$.

[4] R. Dipankar, K. Nagaraja and A. Venkataramani, "J. ACM SIGMOBILE Mobile Computing and Communications Review", Mobility First: a robust and trustworthy mobility-centric architecture for the future internet, vol. 16, no. 2, (2012).

[5] D. Miorandi, S. Sicari, F. De Pellegrini and I. Chlamtac, "J. Ad Hoc Networks. Internet of things: Vision, applications and research challenges", vol. 10, no. 1497, (2012).

[6] F. Akyildiz, D. Pompili and T. Melodia, "J. ACM SIGbed Review. Challenges for efficient communication in underwater acoustic sensor networks", vol. 1, no. 3, (2004).

[7] F. Akyildiz, D. Pompili and T. Melodia, "J. Ad Hoc Networks. Underwater acoustic sensor networks: research challenges", vol. 3, no. 257, (2005).

[8] R. Diamant, G. N. Shirazi and L. Lampe, "Robust Spatial Reuse Scheduling in Underwater Acoustic Communication Networks", 74th Vehicular Technology Conference, (2011) September.

[9] L. Freitag and M. Stojanovic, "Acoustic communications for regional undersea observatories", Proceedings of Oceanology International, (2002) March, UK, London.

[10] Jain, K. Fall and R. Patra, "Routing in a Delay Tolerant Network", Proceedings of ACM SIGCOMM, (2004).

[11] T. Vu, A. Baid, Y. Zhang, T. D. Nguyen, J. Fukuyama, R. P. Martin and D. Raychaudhuri-'DMap: A Shared Hosting Scheme for Dynamic Identifier to Locator Mappings in the GlobaA Internet', IEEE 32nd International Conference (ICDCS), (2012) June.

[12] N. Randazzo and C. Bechaz, "Scientific application of advanced undetwater positioning techniques", In Oceans IEEE, (2005), Europe.

[13] K. Fall, "A delay-tolerant network architecture for challenged internets", Proceedings Conference on Applications, technologies, architectures, and protocols for communications, (2003).

[14] S. Nelson, G. Bhanage and D. Raychaudhuri, GSTAR: Generalized Storage-Aware Routing for Mobility First in the Future Internet Architecture" Proceedings 6f the sixth international workshop on MobiArch, (2011) June.

[15] O. Saleh and M. Hefeeda, "Modeling and caching of peer-topeer traffic", Proceedings of the 2006 IEEE International Conference on Network Protocols, (2006), USA, Washington.

[16] Y. Sun and T. Melodia, "The Internet Underwater, An IP-compatible Protocol Stack for Commercial Undersea Modems", Proceedings of the eigth ACM infernational Conference on Underwater Networks and Systems, (2013) November.

[17] Z. Zhang, S.-L. Lin and K. T. Sung, "A Prediclion-Based Delay-Tolerant Protocol for Underwater Wireless Sensor Networks", Whreless Communications and Signal Processing, (2010).

[18] K. Muppalla, N.-Y. Yun, S.-H. Park and C.Kim, "Design of Underwater Things and IoUT Architecture using Delay Tolerant Network", The 2014 International conference on Green and Human Information Technology ICGHIT, (2014) Febpuary. Ho Chi Minh City, Vietnam

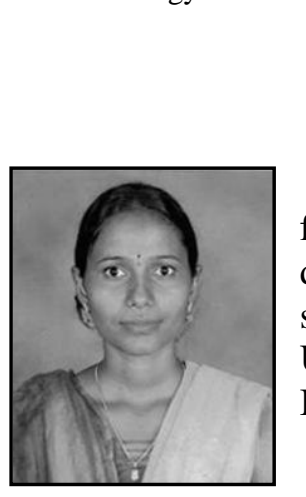

\section{Authors}

Muppalla Kalyani, She has received her Bachelor degree in 2009 from Sri Venkateswara University, India and received Master's degree in 2012 from JNTU Anantapur University, India. Currently she is Ph.D. student in Graduate School of Business IT, Kookmin University, Korea. Her current research interests include Underwater IoT (Internet of Things) and Ubiquitous Network.

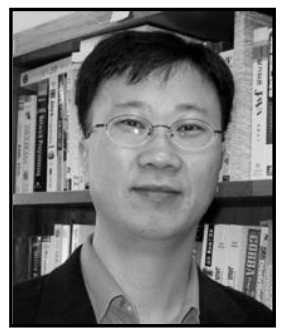

Soo-Hyun Park, He has received his B.S., M.S. and Ph. D degrees in computer science engineering from Korea University in 1988, 1990 and 1998, respectively. Now, he is a professor in the Department of Information System, Kookmin University, Korea. His current research interests include Underwater IoT (Internet of Things) and Ubiquitous Network. 


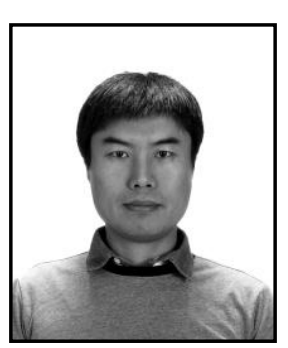

Nam-Yeol Yun, He has received his $\mathrm{M}$. Sc and $\mathrm{Ph}$. D degrees from Kookmin University, Seoul, Korea in 2009 and 2014, respectively. Now, he is researcher in Ocean Sensor Network Research Center, Korea. His current research interests include MAC protocols and implementation of wireless communication and acoustic communication systems and embedded software in Underwater Acoustic Sensor Networks (UWASNs).

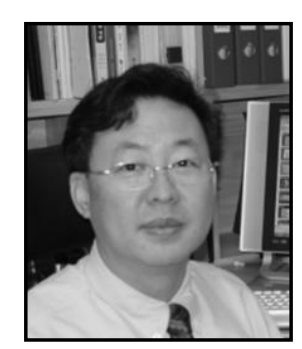

Changhwa Kim, He has received his master's degree and $\mathrm{Ph}$. D degrees in computer science from Korea University in 1987 and 1990, respectively. Now, he is a professor in the Department of Computer Science and Engineering, Gangneung National Universjuty Korea. His current research interests include Underwater Iot (linternet of Things), Ubiquitous Sensor Networks and Distributed System.

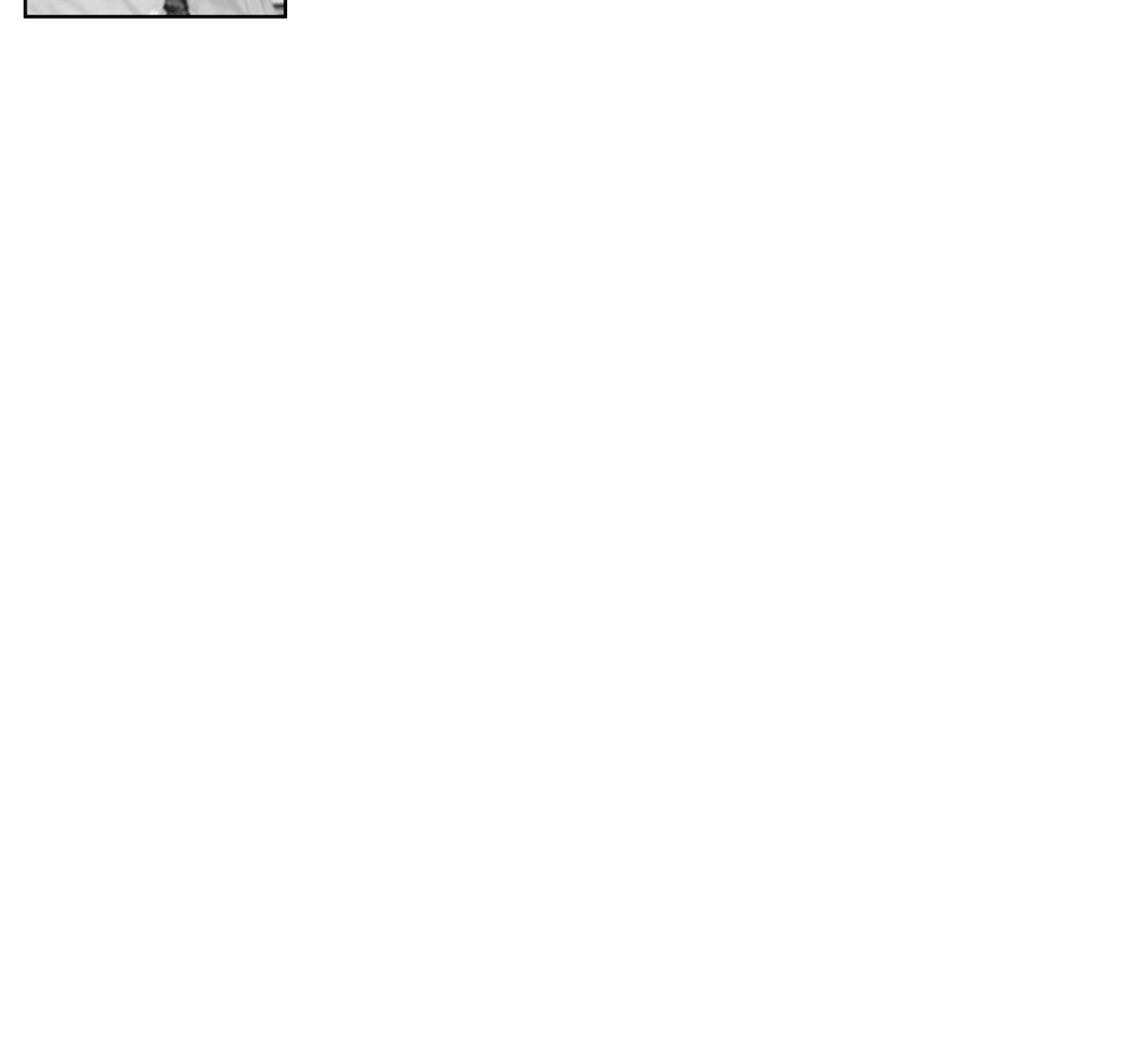

